\title{
Scalar graviton as dark matter
}

\author{
Yu. F. Pirogov \\ Theory Division, Institute for High Energy Physics, Protvino, Moscow Region, Russia
}

\begin{abstract}
In the report, the theory of unimodular bimode gravity built on principles of unimodular gauge invariance/relativity and general covariance is exposed. Besides the massless tensor graviton of General Relativity, the theory includes an (almost) massless scalar graviton treated as the gravitational dark matter. A spherically symmetric vacuum solution, describing the coherent scalar-graviton field for the soft-core dark halos with the asymptotically flat rotation curves, is demonstrated.
\end{abstract}

\section{Introduction}

The report presents an extension to General Relativity (GR) based on the unimodular relativity and general covariance [1]-6], the so-called Unimodular Bimode Gravity (UBG) 1 The basic principles of the theory and the simplified Lagrangian model are indicated. The dark halos built of a new particle - the (nearly) massless scalar graviton are shown to naturally emulate the galaxy dark matter (DM) halos.

\section{Unimodular relativity}

Gauge invariance/relativity To be consistent as the effective field theory, in particular, to allow for the quantum corrections, a gravity theory, describing the spin-two field, should be built on a gauge principle. The essence of the theory is determined by its gauge properties under the diffeomorphism transformations, and the group of the gauge invariance/relativity. More particularly, the diffeomorphisms are given by the coordinate transformations: $x^{\mu} \rightarrow x^{\prime \mu}=x^{\prime \mu}(x)$ followed by the field substitutions $\varphi(x) \rightarrow \varphi^{\prime}\left(x^{\prime}\right)$. At that, the local properties if the theory are determined by the infinitesimal diffeomorphims: $x^{\mu} \rightarrow x^{\prime \mu}=x^{\mu}+\delta_{\xi} x^{\mu}$, with $\delta_{\xi} x^{\mu} \equiv-\xi^{\mu}$ being a vector field. These properties are expressed through the so-called Lie derivative given by the net infinitesimal variation of a field exclusively due to its tensor properties: $\delta_{\xi} \varphi(x)=\left(\varphi^{\prime}\left(x^{\prime}\right)-\varphi\left(x^{\prime}\right)\right)_{x^{\prime} \rightarrow x}$. In particular, one has for metric

$$
\begin{aligned}
\delta_{\xi} g_{\mu \nu} & =g_{\mu \lambda} \partial_{\nu} \xi^{\lambda}+g_{\nu \lambda} \partial^{\lambda} \xi_{\mu}+g_{\mu \nu} \partial_{\lambda} \xi^{\lambda} \\
& =\nabla_{\mu} \xi_{\nu}+\nabla_{\nu} \xi_{\mu},
\end{aligned}
$$

where $\xi_{\mu}=g_{\mu \nu} \xi^{\nu}$ and $\nabla_{\mu}$ is a covariant derivative. For a scalar field one has $\delta_{\xi} \phi=$ $\xi^{\mu} \partial_{\mu} \phi$. The general gauge invariance/relativity corresponds to the group of the general diffeomorphisms (GDiff):

GDiff : $\xi^{\mu}$ unrestricted.

\footnotetext{
${ }^{1}$ For a self-contained exposition, see [6].
} 
By means of GDiff, one can eliminate in metric all the excessive degrees of freedom, but for two corresponding to the transverse-tensor graviton. As a result, this ensures the masslessness of the graviton, $m_{g}=0$. This takes place in GR, as well as in its generally invariant extensions.

Of particular interest is the field $\sqrt{-g}, g \equiv \operatorname{det}\left(g_{\mu \nu}\right)$, which may, under certain conditions, present an additional, scalar degree of freedom in metric. The respective Lie derivative is

$$
\delta_{\xi} \sqrt{-g}=\frac{1}{2} \sqrt{-g} g^{\mu \nu} \delta_{\xi} g_{\mu \nu}=\partial_{\mu}\left(\sqrt{-g} \xi^{\mu}\right)
$$

or otherwise

$$
\delta_{\xi} \ln \sqrt{-g}=\xi^{\mu} \partial_{\mu} \ln \sqrt{-g}+\partial_{\mu} \xi^{\mu} .
$$

Define the group of the transverse diffeomorphisms (TDiff) as

$$
\text { TDiff : } \partial_{\mu} \xi^{\mu}=0 \text {. }
$$

It proves, that TDiff is necessary and sufficient for $m_{g}=0$ [7], with GDiff of GR being, in fact, overabundant for this purpose. Moreover, it is seen that under TDiff the quantity $\ln \sqrt{-g}$ behaves like a scalar field and may thus be used as an extra degree of freedom in metric, not spoiling the masslessness of the (tensor) graviton. The metric theory of gravity built on the transverse gauge invariance/relativity is the so-called Transverse Gravity (TG) (see, e.g., [8]). However, at face value, such a theory is not generally covariant. To remedy this introduce a nondynamical scalar density $\bar{\mu}$ (the so-called modulus) transforming as $\sqrt{-g}$, so that $\varsigma \equiv \ln (\sqrt{-g} / \bar{\mu})$ behaves like a scalar field under GDiff. Define then the group of the unimodular diffeomorphisms (UDiff) as preserving the modulus:

$$
\text { UDiff : } \delta_{\xi} \bar{\mu}=\partial_{\mu}\left(\bar{\mu} \xi^{\mu}\right)=0 \text {, }
$$

The theory of gravity built on the unimodular invariance/relativity is Unimodular Gravity (UG). Being generally covariant, $\mathrm{UG}$ is equivalent to the non generally covariant TG in coordinates where $\bar{\mu}=1$ (if any). But now due to the general covariance, one can use GDiff to study the theory in the arbitrary observer's coordinates.

Physical/helicity content Indicate the physical/helicity content of UG. Consider the weak-gravity-field approximation: $g_{\mu \nu}=\eta_{\mu \nu}+h_{\mu \nu}, \sqrt{-g}=1+h / 2$, with $h=\eta^{\mu \nu} h_{\mu \nu}=$ $h_{00}-h_{l l}$. Under GDiff, one has

$$
\begin{aligned}
& \delta_{\xi} h=\xi^{\mu} \partial_{\mu} h+2 \partial_{\mu} \xi^{\mu}, \\
& \delta_{\xi} \bar{h}=\xi^{\mu} \partial_{\mu} \bar{h}+2 \partial_{\mu} \xi^{\mu},
\end{aligned}
$$

where $\bar{h} / 2 \equiv \ln \bar{\mu}$ (not necessarily small). This means that $h$ and $\bar{h}$ separately can be eliminated due to GDiff. Nevertheless, $\varsigma=(h-\bar{h}) / 2$ transforming homogeneously as a scalar field can not be removed and may thus serve as an independent degree of freedom. With the rest of components in $h_{\mu \nu}$ transforming under GDiff exactly as in GR, the physical/helicity content of UG thus includes:

- transverse deformation (4-volume preserving)/tensor graviton/graviton $\left(\lambda_{g}= \pm 2\right)$;

- compression (form preserving) mode/scalar graviton/systolon $\left(\lambda_{s}=0\right)$.

The bimode gravity mediated by the systolon and graviton may naturally be referred to as the systo-gravity. 


\section{General covariance}

Unimodular Gravity To be precise, under the Unimodular Gravity (UG) we understand collectively any generally covariant and unimodular invariant metric theory of gravity containing a nondynamical scalar density (modulus) $\bar{\mu}$. For general covariance, $\bar{\mu}$ may enter only through $\bar{\mu} / \sqrt{-g}$. The UG action in the vacuum looks generically like:

$$
S=\int \mathcal{L} d^{4} x=\int L\left(g_{\mu \nu}, \bar{\mu} / \sqrt{-g}\right) \sqrt{-g} d^{4} x,
$$

with $L$ being the scalar Lagrangian. The UG field equations (FEs) in vacuum are obtained by extremizing $S$ under $\delta \bar{\mu}=0$, with the result

$$
\mathcal{G}^{\mu \nu} \equiv \delta \mathcal{L} / \delta g_{\mu \nu}=0
$$

and $\bar{\mu}$ appearing as an external functional parameter. Ultimately, this signifies the openness of the system of fields considered and may, conceivably, serve as an off-spring of a "new physics". Some particular cases of UG are as follows.

General Relativity The marginal case of with the absence of $\bar{\mu}$ corresponds to GR:

$$
\begin{aligned}
\mathcal{L}= & -\kappa_{g}^{2}\left(\frac{1}{2} R-\Lambda\right) \sqrt{-g} \\
& R^{\mu \nu}-\frac{1}{2} R g^{\mu \nu}+\Lambda g^{\mu \nu}=0,
\end{aligned}
$$

where $\kappa_{g}$ is the Planck mass and $\Lambda$ is a cosmological constant. The GR vacuum corresponds thus to $R=4 \Lambda$.

Unimodular Relativity The restriction $\sqrt{-g} / \bar{\mu}=1$ corresponds to the so-called Unimodular Relativity (UR) [9]:

$$
\begin{gathered}
\mathcal{L}=-\left.\frac{\kappa_{g}^{2}}{2}(R \sqrt{-g})\right|_{\sqrt{-g}=\bar{\mu}}, \\
R^{\mu \nu}-\frac{1}{4} R g^{\mu \nu}=0
\end{gathered}
$$

so that $R$ remains undetermined by FEs. The Bianchi identity gives additionally $\partial_{\mu} R=0$. Henceforth, the UR vacuum corresponds to $R=\Lambda_{0}$, with $\Lambda_{0}$ being an integration constant, reproducing effectively the Lagrangian $\Lambda$-term. Thus UR is classically equivalent to GR with a cosmological constant, both theories describing only massles tensor graviton.

Unimodular Bimode Gravity The radically new gravity theory appears when $\bar{\mu} / \sqrt{-g}$ enters through the additional kinetic term. This case corresponds to Unimodular Bimode Gravity (UBG/systo-gravity) [6]:

$$
\begin{aligned}
\mathcal{L} & =\left(-\frac{\kappa_{g}^{2}}{2} R+\frac{\kappa_{s}^{2}}{2} g^{\mu \nu} \partial_{\mu} \varsigma \partial_{\nu} \varsigma\right) \sqrt{-g} \\
\varsigma & \equiv \ln \sqrt{-g} / \bar{\mu}
\end{aligned}
$$

In addition to the massless transverse-tensor graviton, UBG/systo-gravity describes the (nearly) massless scalar graviton/systolon $\varsigma$, characterized by the appropriate mass scale 
$\kappa_{s}<\kappa_{g}$. The UBG/systo-gravity Lagrangian is unique because:

- the higher derivative terms with $\varsigma$ are suppressed by the powers of $1 / \kappa_{s}$;

- the derivativeless terms with $\varsigma$ are forbidden under the global symmetry $\varsigma \rightarrow \varsigma+\varsigma_{0} 2^{2}$

The FEs for UBG/systo-gravity are

$$
R^{\mu \nu}-\frac{1}{2} g^{\mu \nu}-\kappa_{g}^{-2} T_{s}^{\mu \nu}=0
$$

where $T_{s \mu \nu}$ is the effective systolon energy-momentum tensor:

$$
T_{s \mu \nu}=\left(\partial_{\mu} \sigma \partial_{\nu} \sigma-\frac{1}{2} \partial \sigma \cdot \partial \sigma g_{\mu \nu}\right)+\kappa_{s} \nabla \cdot \nabla \sigma g_{\mu \nu},
$$

where $\sigma \equiv \kappa_{s} \varsigma$, The non-harmonic term $\kappa_{s} \nabla \cdot \nabla \sigma$ is specific to UBG/systo-gravity and may serve as a signature of the latter, being absent in other gravity theories such as, e.g.: - GR with a free massless scalar field;

- the free Brans-Dicke theory in Einstein frame.

In essence, such a term, ensuring a peculiar dark halo solution, is in charge for treating the systolon as a DM particle.

\section{Dark halos}

Harmonic scalar field In the case $\nabla \cdot \nabla \sigma=0$, the UBG/systo-gravity FEs are similar to those of GR with a free massless scalar field. Having singularity at the origin, their solutions describe the so-called dark holes/fractures consisting of the scalar field $\sigma$ and the matter at the origin. The particular cases of the dark holes/fractures are:

- the black holes: no scalar field;

- the vacuum holes: no matter 3

Non-harmonic scalar field In the case $\nabla \cdot \nabla \sigma \neq 0$, there appears a principally new spherically symmetric regular solution to the vacuum FEs. It looks approximately like (Fig. 1):

$$
\varsigma_{h}= \begin{cases}\tau^{2}-\frac{3}{10} \tau^{4}+\mathcal{O}\left(\tau^{6}\right), & \tau \leq 1 \\ \ln 3 \tau^{2}, & \tau \gg 1\end{cases}
$$

where $\tau=r / R_{0}$ is the scaled radial distance, with $R_{0}$ being an integration constant. This solution describes the dark halos, with $R_{0}$ being their characteristic distance scales. The energy density profile of a dark halo is as follows (Fig. 2):

$$
\rho_{h} / \rho_{0}= \begin{cases}1-\tau^{2}+\frac{4}{5} \tau^{4}+\mathcal{O}\left(\tau^{6}\right), & \tau \leq 1, \\ 1 /\left(3 \tau^{2}\right)+\mathcal{O}\left(1 / \tau^{5 / 2}\right), & \tau \gg 1\end{cases}
$$

with the central energy density $\rho_{0}=3 \kappa_{s}^{2} / R_{0}^{2}$. This profile lies somewhere in between the two commonly accepted Monte Carlo cases:

- the pseudo-isothermal sphere: $\rho_{\text {ref }} / \rho_{0}=1 /\left(1+\tau^{2}\right)$ (dotted line);

- the isothermal sphere: $\bar{\rho} / \rho_{0}=1 /\left(3 \tau^{2}\right)$ (thin line).

\footnotetext{
${ }^{2}$ For potential $V_{s}(\varsigma)$ violating the global symmetry and producing, in particular, the mass for $\varsigma$, see [4, 6].

${ }^{3}$ For more detail on the dark holes/fractures, in particular, on the difference between their physical interpretation in UBG/systo-gravity and GR with a scalar field, see [3, 6].
} 


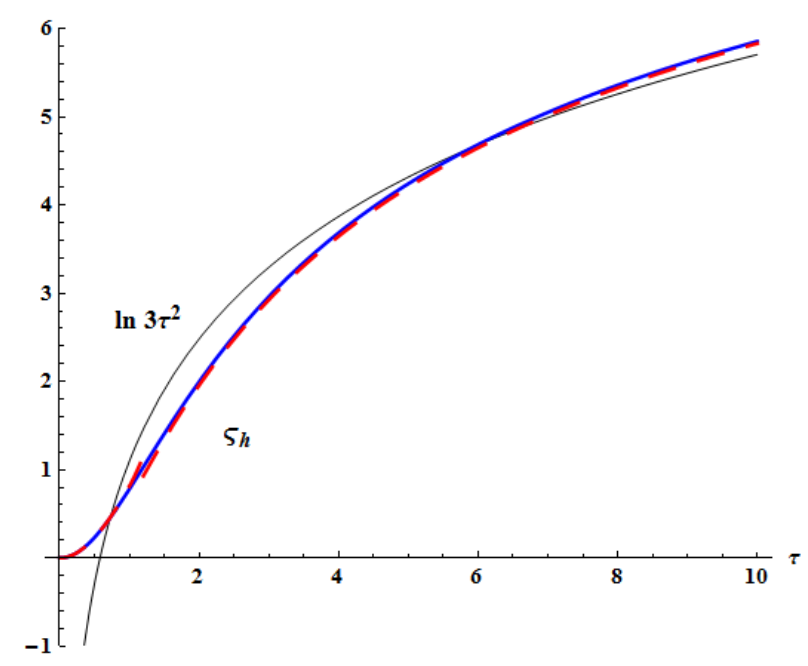

Figure 1: Dark halo solution $\varsigma_{h}(\tau)$.

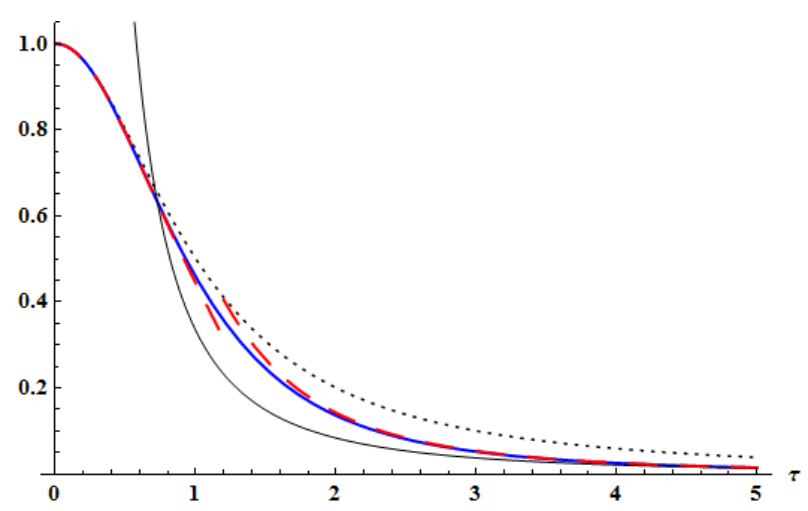

Figure 2: Normalized energy density profile $\rho_{h}(\tau) / \rho_{0}$.

In reality, there should also be included corrections to the halo core due to:

- luminous matter $\rho_{l m}$;

- particle DM $\rho_{d m}$.

The halo rotation curve profile is as follows (Fig. 3):

$$
v_{h}^{2} / v_{\infty}^{2}= \begin{cases}\tau^{2}-\frac{3}{5} \tau^{4}+\mathcal{O}\left(\tau^{6}\right), & \tau \leq 1 \\ 1+\mathcal{O}(1 / \sqrt{\tau}), & \tau \gg 1\end{cases}
$$

with $v_{\infty}$ being the asymptotic rotation velocity: $v_{\infty}=\kappa_{s} / \sqrt{2} \kappa_{g}$. In order for the dark halos to have baring to galaxies, $v_{\infty}$ should be about $10^{-3}$. This approximately fixes the mass scale for the scalar gravity relative to the tensor one.

Altogether, one may say about the coherent dark halos that:

- in the vacuum, they are not only possible but are, in a sense, "predictable", being thus a signature of UBG/systo-gravity (similar to BHs being a signature of GR and its extensions);

- in the presence of matter, they are likely to reflect only the universal long-range tail, with the specific short-range core influenced by matter (including, possibly, the particle DM besides the luminous one); 


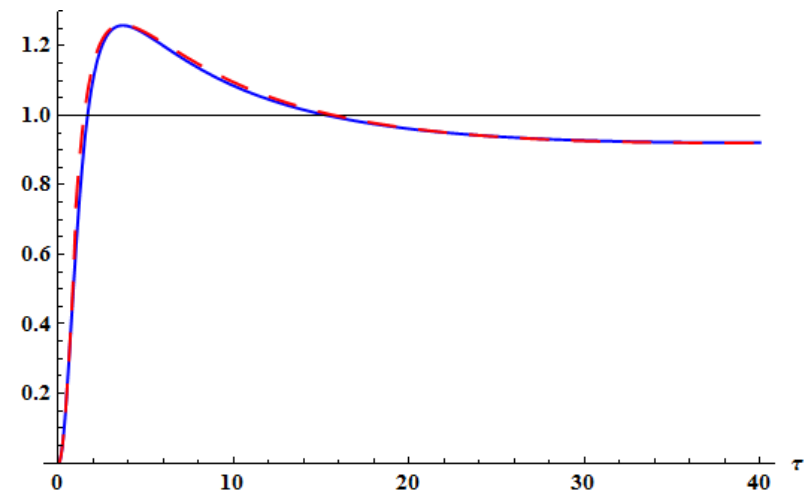

Figure 3: Normalized rotation curve profile $v_{h}(\tau) / v_{\infty}$.

- the two-component DM (the coherent systolon field plus the continuous medium) may eventually present the long-waited solution to the DM puzzle.4

\section{Conclusion}

We conclude as follows:

- UBG/systo-gravity is the theoretically viable extension to GR, safely preserving its solid features, as well as predicting a lot of new phenomena beyond GR;

- the emerging new particle - the (almost) massless scalar graviton/systolon - reveals the properties imminent to DM;

- further studying the theoretical and observational aspects of the theory, especially due to inclusion of matter, is quite urgent.

\section{References}

[1] Yu. F. Pirogov, Phys. Atom. Nucl. 69, 1338 (2006); arXiv:gr-qc/0505031.

[2] Yu. F. Pirogov, arXiv:gr-qc/0609103.

[3] Yu. F. Pirogov, Phys. Atom. Nucl. 73, 134 (2010); arXiv:0903.2018[gr-qc].

[4] Yu. F. Pirogov, Mod. Phys. Lett. A 24, 3239 (2009); arXiv:0909.3311 [gr-qc].

[5] Yu. F. Pirogov and I. Yu. Polev, arXiv:1010.3431 [gr-qc].

[6] Yu. F. Pirogov, Eur. Phys. J. C 72 (2012) 2017; arXiv:1111.1437 [gr-qc].

[7] J. J. van der Bij, H. van Dam and Y. J. Ng, Physica A 116, 307 (1982).

[8] E. Alvarez et. al., Nucl. Phys. B 756, 148 (2006); arXiv:hep-th/0606019.

[9] J. L. Anderson and D. R. Finkelstein, Am. J. Phys. 39, 901 (1971).

\footnotetext{
${ }^{4}$ For more detail on the dark halos, see [4]-6]. Besides, see [6] for the interpolating dark holehalo solutions describing the so-called dark lacunas, which may model the galaxies in toto (but for the distributed matter).
} 